\title{
Simulação termodinâmica computacional de liga modelo de aço super ferrítico contendo $0,07 \%$ de boro
}

\author{
Computational and thermodynamic \\ simulation of a model super-ferritic \\ steel containing $0.07 \%$ wt. of boron
}

Julianne Ribeiro dos Santos ${ }^{1}$, Marcelo José Gomes da Silva ${ }^{1}$, Hamilton Ferreira Gomes de Abreu ${ }^{1}$

\author{
${ }^{1}$ Laboratório de Caracterização de Materiais, Lacam, DEMM/CT/UFC, Campus do Pici, bloco 729, CEP: 60440- \\ 900, Ceará, Fortaleza, Brasil. \\ e-mail: rsjulianne@yahoo.com.br
}

\section{RESUMO}

Aços inoxidáveis super ferríticos são utilizados em equipamentos como trocadores de calor e torres de destilação que operam em condições extremas de temperatura, pressão e em ambientes contendo altas concentrações de cloretos, $\mathrm{H}_{2} \mathrm{~S}$ e $\mathrm{CO}_{2}$, encontradas nas novas áreas de exploração de petróleo e gás na costa brasileira. Uma liga super ferrítica com composição baseada em aços comerciais foi desenvolvida para a realização do presente estudo que visa adaptar a composição dos aços super ferríticos, com o objetivo de aprimorá-los. A liga desenvolvida contem 4,4\% p de níquel (Ni) para melhorar a sua tenacidade, $25,7 \%$ p de cromo $(\mathrm{Cr})$ para que a estabilidade da fase ferrítica fosse mantida devido a adição de Ni, 4,6\%p de Mo para aumentar a resistência à corrosão. O diferencial para o estudo desta liga foi a adição de $0,07 \%$ p (700 ppm) de boro, visando o aumento da temperabilidade, tenacidade e estabilidade de contornos de grão. Foram realizados cálculos termodinâmicos através do programa computacional Thermo-Calc para a simulação dos diagramas de fase e a fração em peso das fases presentes no material. Os resultados das composições experimentais foram comparados com outros trabalhos já realizados com o aço comercial UNS S44660. Através dos resultados obtidos uma estimativa da temperatura de estabilidade da fase ferrítica foi determinada e as possíveis fases deletérias foram identificadas de acordo com variação da temperatura e da composição química.

Palavras-chave: Aço inoxidável super ferrítico, Termonidâmica computacional, Boro.

\begin{abstract}
Super ferritic stainless steels are used in equipment such as heat exchangers and distillation towers operating under extreme temperature and pressure conditions and in environments containing high concentrations of chlorides, $\mathrm{H}_{2} \mathrm{~S}$ and $\mathrm{CO}_{2}$ found in the new areas of oil and gas exploration on the Brazilian coast. A super ferritic alloy with composition based on commercial steels was developed for the present study, aiming to adapt the composition of super ferritic steels in order to improve them. The developed alloy contains 4.4 wt.\% of nickel $(\mathrm{Ni})$ to improve toughness, $25.7 \mathrm{wt}$ \% of chromium $(\mathrm{Cr})$ so that the stability of the ferritic phase is maintained due to the addition of $\mathrm{Ni}, 4.6 \mathrm{wt} . \%$ of Mo to increase the corrosion resistance. The differential for the study of this alloy was the addition of $0.07 \mathrm{wt} . \%$ (700 ppm) of boron, aiming to increase hardenability, toughness and grain boundary stabilization. Thermodynamic calculations were performed using the Thermo-Calc software for the simulation of phase diagrams and the weight fraction of the phases present in the material. The results of the experimental compositions were compared with results from commercial steel UNS S44660. The results obtained an estimate of the stability temperature of the ferritic phase and determined the possible deleterious phases according to temperature variation and chemical composition.
\end{abstract}

Keywords: Super ferritic stainless steel, Computational thermodynamics, Boron.

\section{INTRODUÇÃO}


Os aços inoxidáveis ferríticos são largamente utilizados na indústria em geral, tais como: indústria química, petroquímica, geração de energia, bombas, implantes e instrumentos cirúrgicos além de uma infinidade de outros produtos de uso doméstico e comercial, por possuírem boas propriedades mecânicas e boa resistência à corrosão SMITH [1]. O aumento do desempenho dos aços inoxidáveis ferríticos é conseguido com teores de $\mathrm{Cr}$ acima de $25 \%$ e adições de Mo que melhoram a resistência à corrosão em diversos meios. Alguns trabalhos afirmam que o aumento do teor de Mo na composição dos aços pode torná-los uma alternativa em aplicações onde compostos naftênicos oriundos de petróleo pesado estejam presentes BAPTISTA et al [2], GALLO e EDMONDSON [3], NEGREIROS et al [4], VASCONCELOS et al [5]. A adição do Mo, no entanto, provoca uma maior precipitação de fases deletérias, tais como fase sigma $(\sigma)$, fase chi $(\chi)$, fase $\mathrm{Mu}(\mu)$, nitretos como $\mathrm{Fe}_{5} \mathrm{Mo}_{13} \mathrm{~N}_{4}$, carbonetos do tipo $(\mathrm{Cr}, \mathrm{Fe}, \mathrm{Mo}) \mathrm{M}_{23} \mathrm{C}_{6}$ ou carbonitretos de $\mathrm{Nb}$ e $\mathrm{Ti}$ como $\mathrm{Nb}(\mathrm{CN})$ e Ti $(\mathrm{CN})$. A presença destas fases pode ter como consequências a fragilização com perda de resistência à corrosão em temperaturas elevadas e a redução da tenacidade ANDRADE et al [6], GUIMARÃES, [7], MOURA et al [8], PARK et al [9].

$\mathrm{Na}$ indústria há um crescente incentivo à pesquisa relacionada à utilização de elementos químicos com baixo custo, dentre eles o boro que é utilizado para aumentar a temperabilidade dos aços. Devido a essa aplicação, tal elemento é considerado um elemento de liga potencial desde meados de 1907. Desde então, a adição de boro tem desempenhado um papel importante no desenvolvimento de aços tratados termicamente DJAHAZI [10], TAYLOR [11], MUN et al [12].

A escassez e o alto custo de muitos dos elementos convencionais (como níquel, cromo e molibdênio) utilizados para o aumento de temperabilidade, criaram um incentivo adicional para o uso de boro como uma alternativa para materiais estratégicos DJAHAZI [10].

Aços com baixo ou médio teor de carbono e com adições de boro, aços ao boro temperados e revenidos têm sido utilizados para diferentes aplicações, como indústria automotiva, de construção, de petróleo, de agricultura, de transportes e na fabricação de plataformas marítimas e tubulações SUSKI, OLIVEIRA [13], LOPEZ-CHIPRES [14]. Depois de vários estudos, foi reconhecido que a adição de pequenas quantidades de boro era suficiente para produzir um efeito significativo nas propriedades destes aços, combinando boa dureza e ductilidade com aumento de propriedades mecânicas quando submetida a tratamentos térmicos DJAHAZI [10], GARLIPP et al [15].

$\mathrm{O}$ boro é um elemento extremamente reativo, reage com oxigênio, formando óxido de boro $\left(\mathrm{B}_{2} \mathrm{O}_{3}\right)$ e com nitrogênio, formando nitreto de boro (BN). Esta fração de boro é denominada boro insolúvel MAVROPOULOS [16].

A primeira dificuldade encontrada na produção de aços ao boro que requerem boa temperabilidade é o fato de ser necessário o controle para assegurar a concentração de boro solúvel no produto final. Em virtude disso, são realizadas algumas práticas de aciaria para prevenir a perda de efetividade do boro pela formação de $\mathrm{B} 2 \mathrm{O} 3$. A adição de boro é realizada depois da adição dos desoxidantes (usualmente manganês, silício e alumínio) e utilizando procedimentos de panela e/ou molde para evitar reoxidação durante o vazamento do aço. Já a perda de efetividade do boro pela formação de BN é prevenida através da combinação de nitrogênio com fortes formadores de nitretos (Ti e Zr, por exemplo) MAVROPOULOS [16]. Como o nitreto de titânio é mais estável que o nitreto de boro, a formação de TiN é favorável, comprovando a eficiência da adição de titânio na proteção do boro da associação com nitrogênio.

A adição de boro nos aços promove a formação de diferentes compostos que serão resultantes da solubilidade de boro nos carbonetos existentes em aços comuns (formando borocarbonetos) ou da formação de boretos, nitretos ou óxidos. Como a quantidade que é adicionada de boro em aços comerciais é pequena, há a formação de outros compostos em menor quantidade do quando comparado aos borocarbonetos.

Os objetivos deste trabalho são: estudar através da termodinâmica computacional a evolução de fases com relação à composição e temperatura e determinar as etapas de processamento térmico que este material será submetido para evitar a precipitação de fases deletérias às propriedades.

\section{MATERIAIS E MÉTODOS}

A Tabela 1 mostra a composição da liga super ferrítica com adição de boro (LSFB) utilizada na simulação computacional. Utilizando o software Thermo-Calc $®$ e a base de dados TCFE6, foram construídos os diagramas de fases da liga com a composição proposta em função da variação da percentagem de Cr. Além disso, o diagrama de fração de peso de cada fase foi construído em função da temperatura. 
Tabela 1: Composição química da liga modelo de aço inoxidável super ferrítico (\% em massa).

\begin{tabular}{c|c|c|c|c|c|c|c|c}
\hline Liga & $\mathbf{C r}$ & Mo & Ni & $\mathbf{C}$ & Mn & Si & B & Fe \\
\hline LSFB & 25,7 & 4,6 & 4,4 & 0,02 & 0,48 & 0,6 & 0,07 & Equil. \\
\hline
\end{tabular}

Fonte: Fabricante

Foi fabricado um lingote com as especificações acima e uma amostra deste lingote foi cortada e preparada metalograficamente através do lixamento até o mesh de 1200 , seguido de polimento em alumina $0,05 \mu \mathrm{m}$ e por fim foi realizado o ataque químico para revelar a microestrutura com behara aquecido a $50{ }^{\circ} \mathrm{C}$ e ácido nítrico $40 \%$. Antes da realização do ataque, a amostra foi submetida a análises de difração de raios-X Philips X-pert Pro com radiação de cobalto $(\lambda=1,79026 \AA ̊)$, e após esta análise a amostra com a microestrutura revelada foi submetida a microscopia ótica Ziess® modelo AX10 acoplado a um computador para equalização das imagens usando o programa AxioCam e microscopia eletrônica de varredura marca Philips, modelo XL-30, equipado com detectores de elétrons secundários (SE - secondary electron) e retroespalhado (BSE - backscattering electron), e de um detector de energia dispersiva de raios-X (EDS) de marca EDAX, para a verificação da existência das fases encontradadas na simulação termodinâmica.

\section{RESULTADOS}

O diagrama de fase para a liga experimental foi construído de acordo com a composição química mostrada na Tabela 1. A Tabela 2 mostra as fases encontradas no diagrama de equilíbrio de fase que foi construido para liga super ferrítica com adição de $0,07 \%$ boro. Neste diagrama encontra-se as possíveis fases precipitadas para a composição escolhida, nos respectivos intervalos de temperaturas de precipitação sem quantificá-las.

Tabela 2: Fases encontradas no diagrama de equilíbrio do pseudo-binário $\mathrm{Fe}-\mathrm{Cr}$ da liga experimental.

\begin{tabular}{c|c}
\hline Intervalo de Temperatura $\left({ }^{\circ} \mathbf{C}\right)$ & Fase \\
\hline $200-450$ & Laves, $\mathrm{M}_{6} \mathrm{C}, \mathrm{Mu}(\mu)$, Austenita, \\
& Sigma, $\mathrm{M}_{23} \mathrm{C}_{6}, \mathrm{M}_{2} \mathrm{~B}$ \\
\hline $451-684$ & Chi $(\chi)$, Austenita, Sigma, $\mathrm{M}_{23} \mathrm{C}_{6}$, \\
& $\mathrm{M}_{2} \mathrm{~B}$ \\
\hline $685-949$ & Austenita, Sigma, $\mathrm{M}_{23} \mathrm{C}_{6}, \mathrm{M}_{2} \mathrm{~B}$ \\
\hline $950-1018$ & $\mathrm{M}_{23} \mathrm{C}_{6}, \mathrm{M}_{2} \mathrm{~B}$ \\
\hline $1018-1245$ & $\mathrm{M}_{2} \mathrm{~B}$ \\
\hline
\end{tabular}

Uma identificação quantitativa das fases pode ser avaliada através da análise dos gráficos da percentagem em peso de cada fase em função da temperatura. Os intervalos de temperaturas nas quais ocorre a precipitação para cada fase também são observados. A Figura 1 apresenta o gráfico de identificação quantitativa da fase Laves. Pode-se observar que a precipitação desta fase ocorre entre $200-325{ }^{\circ} \mathrm{C}$ para a fase Laves e a fração máxima em peso encontrado é de 0,067 .

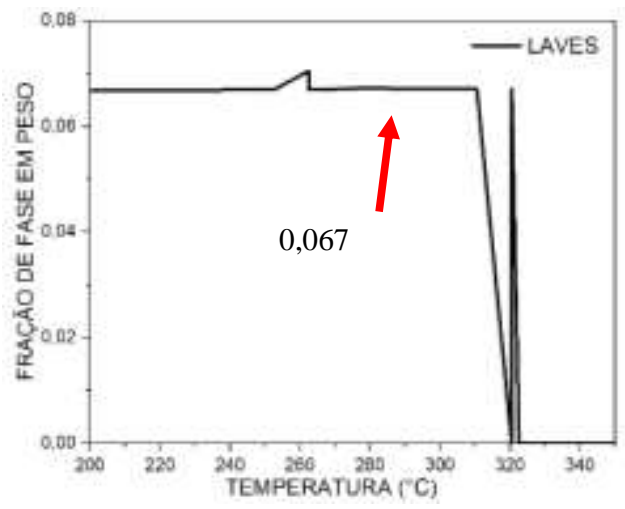

Figura 1: Identificação quantitativa das fases Laves (a). 
A Figura 2 apresenta os gráficos da identificação quantitativa do Carboneto $\left(\mathrm{M}_{6} \mathrm{C}\right)$ e das fases $\mathrm{Mu}$ e Chi, onde podemos observar que o intervalo de temperatura de precipitação destas fases é de $320-343^{\circ} \mathrm{C}$ para o Carboneto $\left(\mathrm{M}_{6} \mathrm{C}\right), 320-410{ }^{\circ} \mathrm{C}$ para a fase $\mathrm{Mu}(\mu)$ e $590-685^{\circ} \mathrm{C}$ para a fase $\mathrm{Chi}(\chi)$. A fração máxima em peso encontrado foi de respectivamente $0,0064,0,042$ e 0,027 .
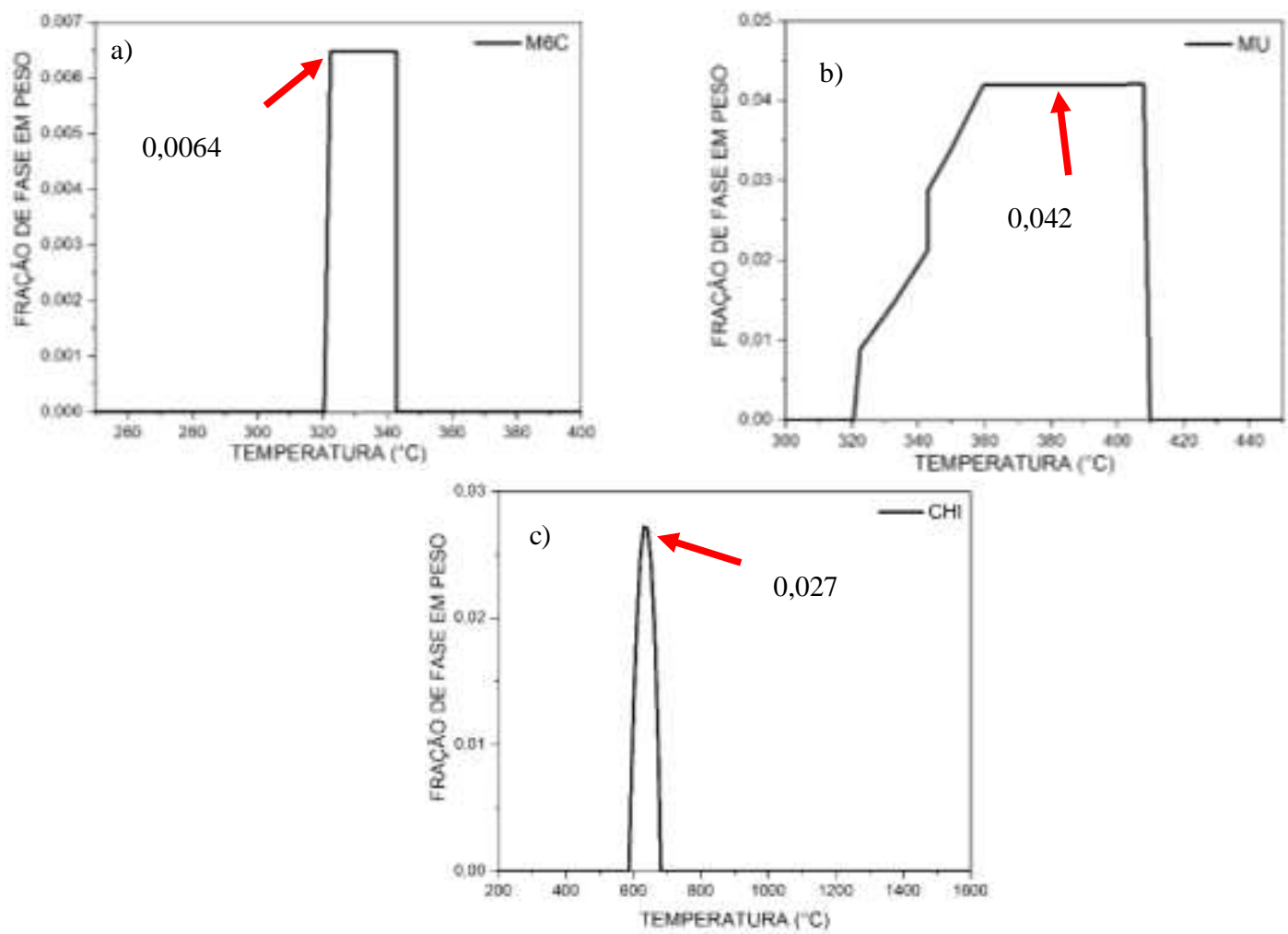

Figura 2: Identificação quantitativa do Carboneto $\left(\mathrm{M}_{6} \mathrm{C}\right)(\mathrm{a})$ e das fases $\mathrm{Mu}(\mu)(\mathrm{b}), \mathrm{Chi}(\chi)(\mathrm{c})$.

A Figura 3 apresenta os gráficos da identificação quantitativa da matriz ferrítica e das fases austenita $(\gamma)$ e sigma $(\sigma)$, onde observar-se que o intervalo de temperatura de precipitação é de $200-940^{\circ} \mathrm{C}$ para a austenita e de $410-950^{\circ} \mathrm{C}$ para a fase sigma, e a fração máxima em peso encontrada é respectivamente 0,41 e 0,37 .
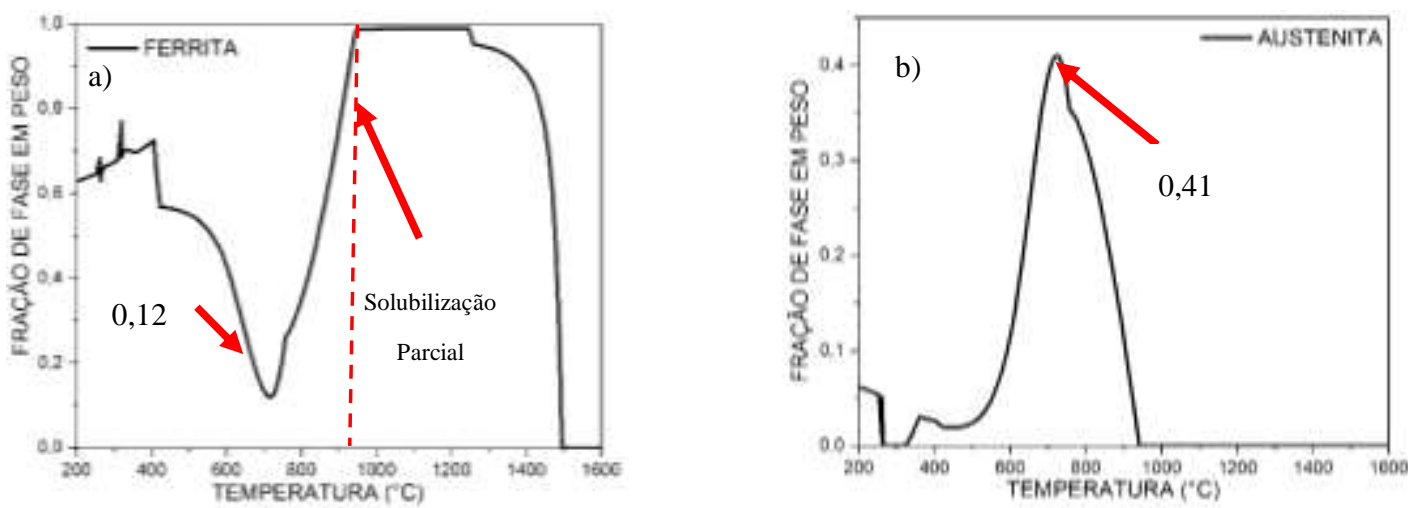


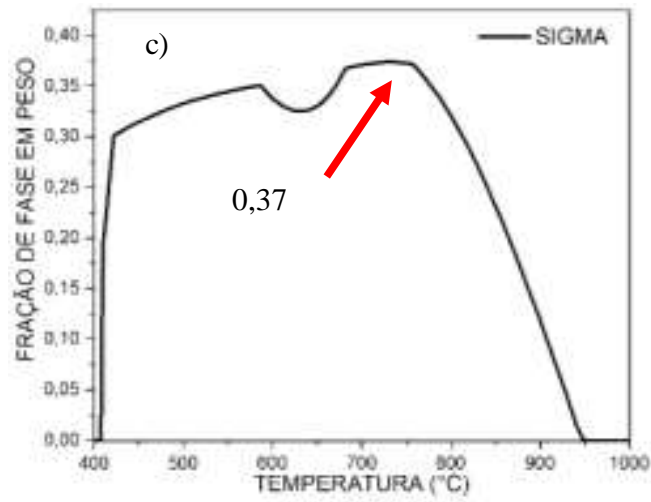

Figura 3: Identificação quantitativa da matriz ferrítica (a), da fase austenita $(\gamma)(b)$ e fase sigma ( $\sigma)(c)$.

Os gráficos apresentados na Figura 4 mostram a identificação quantitativa do carboneto $\left(\mathrm{M}_{23} \mathrm{C}_{6}\right)$ e da fase $\mathrm{M}_{2} \mathrm{~B}$. Podemos observar que ambos precipitados são encontrados em um amplo intervalo de temperatura, o carboneto só será solubilizado à $1020^{\circ} \mathrm{C}$ mantendo um o percentual máximo de aproximadamente de 0,044 e o boreto permanecerá na microestrutura até a temperatura de $1245^{\circ} \mathrm{C}$ com uma fração de 0,0109 .
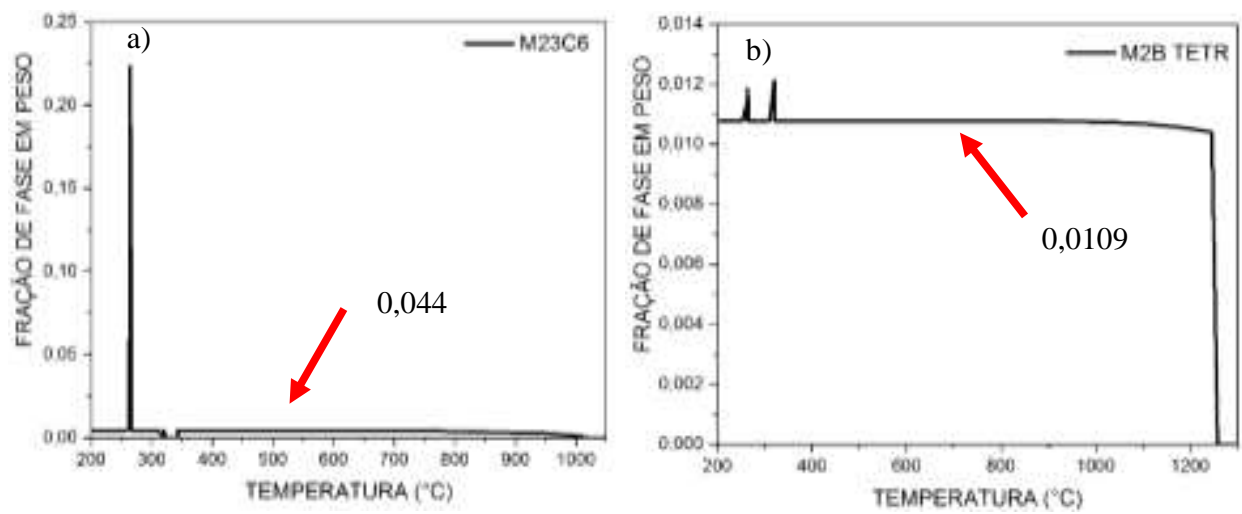

Figura 4: Identificação quantitativa do Carboneto $\left(\mathrm{M}_{23} \mathrm{C}_{6}\right)$ (a) e da fase $\mathrm{M}_{2} \mathrm{~B}$ (b).

O difratograma de raio- $X$ foi obtido buscando identificar as fases encontradas na simulação, com um maior interesse nos borocarbonetos do tipo $\mathrm{M}_{23}(\mathrm{~B}, \mathrm{C})_{6}$ ou $\mathrm{Fe}_{3}(\mathrm{~B}, \mathrm{C})$. O resultado obtido através da técnica de difração de raio-X é apresentado na Figura 5.

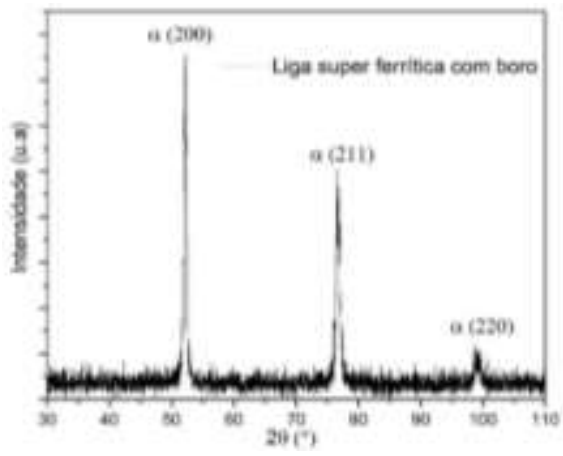

Figura 5: Difratograma de raios-X (tubo de cobalto, $\lambda=1,79026 \AA \hat{)}$ ), para o material laminado a quente e solubilizado com Boro. 
Para a análise da microestrutura foram realizadas microscopias após o preparo metalográfico da amostra. Na Figura 6 (a) é apresentado o resultado da microscopia óptica onde constata-se a presença diferentes fases nucleadas na matriz ferrítica e na Figura 6 (b) é apresentado a microscopia eletrônica de varredura mostrando a existência de fases precipitadas no contorno de grão.
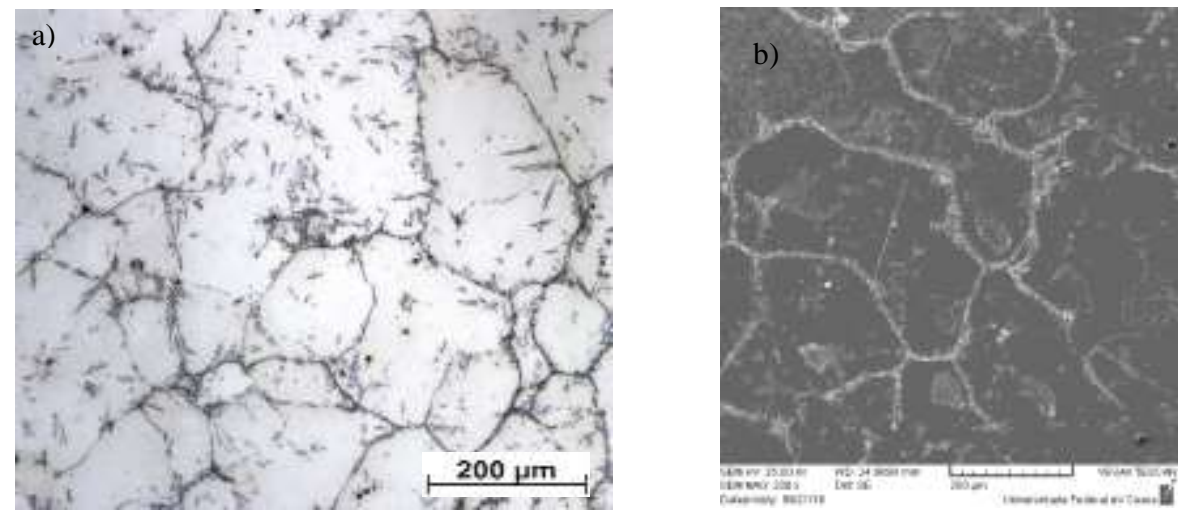

Figura 6: Microestrutura da liga super ferrítica com boro na condição de como recebido (CR) da fundição

A figura 7 apresenta a identificação da fase encontrada no contorno de grão ferrita/austenita, realizada através de análises das composições químicas utilizando à técnica de espectroscopia de energia dispersiva de raios-X ou EDS (Energy Dispersive X-Ray Spectroscopy).

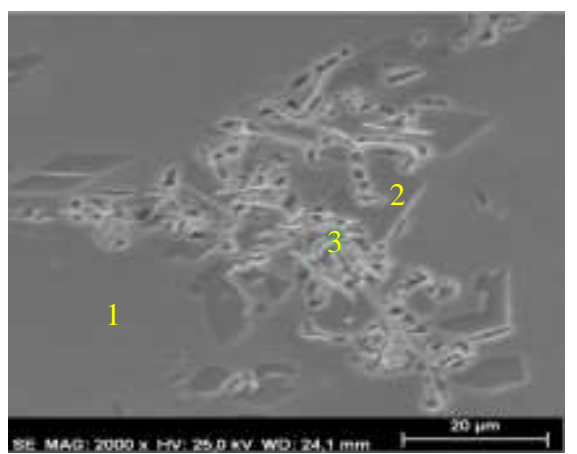

\begin{tabular}{|c|c|c|c|}
\hline $\begin{array}{l}\text { Ele- } \\
\text { mento }\end{array}$ & $\begin{array}{l}(\% \\
1\end{array}$ & $(\% \mathrm{p} / \mathrm{p}) 2$ & $(\% \mathrm{p} / \mathrm{p}$ \\
\hline $\mathrm{Cr}$ & 24,89 & 21,5 & 46,97 \\
\hline $\mathrm{Fe}$ & 64,01 & 67,34 & 44,41 \\
\hline $\mathrm{Ni}$ & 5,07 & 6,55 & 3,01 \\
\hline Mo & 6,04 & 4,61 & 5,61 \\
\hline
\end{tabular}

Figura 7: Análise de EDS na microestrutura da liga super ferrítica com boro na condição de como recebido (CR) da fundição.

\section{DISCUSSÃO}

Com base na simulação realizada é possível observar que a precipitação das fases deletérias Laves, $\mathrm{M}_{6} \mathrm{C}, \mathrm{Mu}$ $(\mu)$, Chi $(\chi)$, sigma $(\sigma)$ e $\mathrm{M}_{23} \mathrm{C}_{6}$ é termodinamicamente favorável para a composição estudada (Figuras de 1 a 4). Estas fases são formadas a partir de reações difusionais e são caracterizadas por serem duras e frágeis. Quando são encontradas na microestrutura do material, podem ser prejudiciais às propriedades mecânicas e de resistência a corrosão, principalmente nos intervalos de tempertaura onde ocorre a precipitação ANDRADE et al [6], GUIMARÃES [7], MOURA et al [8], PARK et al [9].

Nas simulação termodinâmica realizada para a liga modelo foi encontrada uma maior quantidade de fases deletérias do que na simulação obtida para liga comercial UNS S44660, realizada por MOURA et al [18]. Na liga comercial, somente é encontrada a matriz ferrítica, austenita $(\gamma)$ e fase sigma $(\sigma)$. Este fato ocorre devido ao aumento do teor de elementos de liga como Ni e Mo na liga modelo e tais elementos são constituintes nas fases deletérias encontradas na simulação.

Pode-se observar que no intervalo de temperaturas de $600{ }^{\circ} \mathrm{C}$ a $940{ }^{\circ} \mathrm{C}$, há um aumento na precipitação da fase austenita $(\gamma)$ e da fase sigma $(\sigma)$, juntamente com a diminuição considerável da matriz 
ferrítica, que atinge a fração mínima em peso de 0,012 na temperatura de $715{ }^{\circ} \mathrm{C}$, sugerindo que nesta temperatura parte da matriz ferrítica é transformada parcialmente em austenita $(\gamma)$ e em fase sigma $(\sigma)$.

A temperatura acima da qual dissolve-se toda austenita e somente existem a matriz ferrítica e os precipitados na forma de borocarbonetos do tipo $\mathrm{M}_{23}(\mathrm{~B}, \mathrm{C})_{6}$ ou boreto do tipo $\mathrm{Fe}_{2}(\mathrm{~B}, \mathrm{C})$ pode ser determinada a partir do gráfico da identificação quantitativa de fase ferrita em função da temperatura apresentado na Figura 3 (a). A temperatura de solubilização parcial é $949^{\circ} \mathrm{C}$ e pode ser utlizada para promover a homogeneidade da microestrutura.

Por ser extremamente reativo, é necessário o controle da concentração de boro solúvel no produto final, para assegurar para que este material tenha uma boa temperabilidade, tenacidade e a estabilidade dos contornos de grão. De uma maneira geral, o teor de boro solúvel corresponde à quantidade de boro como soluto na matriz (livre ou dissolvido), na forma de borocarbonetos do tipo $\mathrm{M}_{23}(\mathrm{~B}, \mathrm{C})_{6}$ ou boreto do tipo $\mathrm{Fe}_{2}(\mathrm{~B}, \mathrm{C})$. O boro insolúvel corresponde ao teor de boro na forma de nitreto de boro, óxidos e algumas inclusões não metálicas CASARIN [19]. O resultado da simulação termodinâmica mostra que não há a formação de nitretos e óxidos. Mostra também que possivelmente será encontrado boro solúvel na forma de borocarbonetos do tipo $\mathrm{M}_{23}(\mathrm{~B}, \mathrm{C})_{6}$ e do boreto do tipo $\mathrm{M}_{2} \mathrm{~B}$ (Figura 4), logo tais fases foram a de maior interesse para a identificação na microestrutura.

Na Figura 5 pode-se observar que o resultado obtido através da difração de raios-X não foi eficiente para a identificação das fases de interesse $\left(\mathrm{M}_{23}(\mathrm{~B}, \mathrm{C})_{6}\right.$ e $\left.\mathrm{Fe}_{3}(\mathrm{~B}, \mathrm{C})\right)$, visto que os picos obtidos são somente os da matriz ferrítica. Este fato ocorre por limitações da técnica de difração de raios-X que dificilmente detecta fases presentes em frações inferiores a 5\%p. Na Figura 6 (a) constatou-se a presença de austenita (fase clara) nucleada na matriz ferrítica (fase cinza), encontrada principalmente nos contornos de grão ferrita/ferrita. Encontramos também uma terceira fase (fase mais escura) nucleada nos contornos de grão ferrita/austenita que são melhor visualizadas na Figura 6 (b). A austenita encontrada na microestrutura possivelmente é resultado do processo de solidificação do lingote. Durante a solidificação os elementos com menor solubilidade na matriz (fase ferrítica) são segregadas para a região adjacente. No caso o elemento segregado é o Ni, logo, esta região fica enriquecida e há formação da austenita retida nos contornos de grão.

Na Figura 7 observa-se que alguns dos precipitados encontrados no contorno de grão são ricos em cromo e possui um valor de molibdênio maior que o encontrado na matriz da liga, o que sugere por sua composição e morfologia ser a fase sigma. A precipitação desta fase na solidificação deve-se ao fato do cromo e o molibdênio serem insolúveis na austenita, logo, quando a matriz segrega o níquel e há a formação da austenita, a região entre a matriz e a austenita retida fica enriquecida nos elementos cromo e molibdênio favorecendo a precipitação da fase sigma. Estudos mais detalhados para identificação das fases deletérias serão realizadas em pesquisas futuras.

\section{CONCLUSÕES}

1. A composição da liga em estudo favorece a precipitação das fases Laves, $\mathrm{M}_{6} \mathrm{C}, \mathrm{Mu}(\mu)$, Chi $(\chi)$, Sigma $(\sigma)$ e $\mathrm{M}_{23} \mathrm{C}_{6}$.

2. Na simulação termodinâmica realizada foram encontrados borocarbonetos do tipo $\mathrm{M}_{23}(\mathrm{~B}, \mathrm{C})_{6}$ e o boreto do tipo $\mathrm{M}_{2} \mathrm{~B}$ o que indica a provável presença de boro solúvel, importante para a boa temperabilidade da liga e não foi encontrado boro insolúvel, presentes em forma de óxidos e nitretos.

3. Não foi possivel a identificação das fases encontradas na simulação através de difração de raios-X.

4. Através das microscopias realizadas foi possível observar a presença de outras fases na matriz ferrítica, provavelmente austenita e fase sigma.

5. A temperatura de operação ideal deve ser mantida abaixo de $600{ }^{\circ} \mathrm{C}$ para evitar maiores precipitações de austenita e de fase sigma.

6. Para uma solubilização completa da liga em estudo seria necessário uma temperatura superior a temperatura de $1245^{\circ} \mathrm{C}$.

\section{AGRADECIMENTOS}

A Fundação Cearense de Apoio a Pesquisa (Funcap), ao Laboratório de Caracterização de Materiais (Lacam) pelo material e equipamentos fornecidos para a elaboração da presente pesquisa, ao Instituto de Pesquisa Desenvolvimento e Inovação (IPDI) pelas análises realizadas no microscópio eletrônico de varredura. 


\section{BIBLIOGRAFIA}

[1] SMITH,W.F.Structure and properties of engineering alloys. 2nd ed. New York, McGraw-Hill,1993.

[2] BAPTISTA, I.P., JÓIA, C.J.B.M., FONTES, R.G.M., et al., "Sistema e metodologia de avaliação da corrosividade naftênica em laboratório", In: Conferência sobre tecnologia de equipamentos. Proceedings of the 7th COTEQ Conference, 2003.

[3] GALLO, G., EDMONDSON, J. "The effect of molybdenum on stainless steels and naphthenic acid corrosion resistance", In: NACE International, Organizer. Proceedings of the Corrosion Congress, 2008.

[4] NEGREIROS, Y.S., HERCULANO, L.F.G., LIMA-NETO P., et al., "Efeito do teor de Mo na resistência a corrosão de ligas FeCrMo", In: Congresso Brasileiro de Engenharia e Ciência dos Materiais. Proceedings of the 18th CBCiMat Congress. 2008.

[5] VASCONCELOS, I.F., TAVARES, S.S.M., REIS, F.E.U., et al., "Ageing effects on precipitation and resistance to corrosion of a novel Cr-Mo stainless steel with high Mo content", J Mater Sci ., v.44, pp.293299, 2009.

[6] ANDRADE, T.F., KLIAUGA, A.M., PLAUT, R.L., et al., "Precipitation of Laves phase in a $28 \% \mathrm{Cr}-$ $4 \% \mathrm{Ni}-2 \% \mathrm{Mo}-\mathrm{Nb}$ superferritic stainless steel”, Mater Charact., v.59, pp.503-507, 2008.

[7] GUIMARÃES, R.F. Efeito do teor de Mo na microestrutura de juntas soldadas em ligas FeCrMo. Tese de D.Sc.,Fortaleza, Universidade Federal do Ceará, Brasil, 2011.

[8] MOURA, L.B., GUIMARÃES, R.F., ABREU, H.F.G., et al., "Naphthenic corrosion resistance, mechanical properties and microstructure evolution of experimental Cr-Mo steels with Mo content", Mater Res., v. 15, pp. 277-84, 2012. Available from: http://www.scielo.br/pdf/mr/ahead/aop 0942-11.pdf [Internet].

[9] PARK, C.J., AHN, M.K., KWON, H.S. Influences of Mo substitution by W on the precipitation kinetics of secondary phases and the associated localized corrosion and embrittlement in $29 \% \mathrm{Cr}$ ferritic stainless steels. Materials Science and Engineering: A, v. 418, n. 1, p. 211-217, 2006.

[10] DJAHAZI, M. Influence of boron distribution on precipitation and recrystallization in hot worked austenite, Tese de D.Sc., Department of Mining and Metallurgical Engineering, McGill University, Montreal, 1989.

[11] TAYLOR, K.A. Grain-boundary segregation and precipitation of boron in 0.2 percent carbon steels. Metallurgical Transactions A., v. 23A, p. 107-119, 1992.

[12] MUN, D.J. SHIN, E.J. KOO, Y.M. "A study of the behaviour of boron distribution in low carbon steel by particle tracking autoradiography”, Nuclear Engineering anf Technology, v.43, 1, p. 1-6, 2011.

[13] SUSKI, C.A. OLIVEIRA, C.A.S. "Effect of austenitizing temperature on the precipitation of carbides in quenched low carbon boron steels", Metallography, Microstructure, and Anallysis, v.2, n.2, pp.79-87, 2013.

[14] LOPEZ-CHIPRES, E., MEJÍA, I., MALDONADO, C., et al., "Hot ductility behaviour of boron microalloyed steels", Materials and Science Engineering A., v. 460-461, pp. 464-470, 2007.

[15] GARLIPP, W., CILENSE, M., NOVAES G.S.I., "Austenite decomposition of C-Mn steel containing boron by continuous cooling”, Journal of Materials Processing Technology, v. 114, p. 71-74, 2011.

[16] MAVROPOULOS, L.T. The synergistic effect of niobium and boron on recrystallization in hot worked austenite. 1986. Tese de D.Sc., Department of Mining and Metallurgical Engineering, McGill University, 1986.

[17] KOVAK C.W. High performance stainless steels. USA, Nickel Development Institute; 2011. [Cited 2012, February19]. Available from:

http://www.nickelinstitute.org/TechnicalLiterature/Reference\%20Book\%20Series/HighPerformanceStainless Steels 11021.aspx

[18] MOURA L.B., ABREU, H.F.G., NEGREIROS, Y.S. "Computational thermodynamic analysis of secondary phases in super ferritic stainless steels", Journal of Materials Research and Technology, v.2, n.3, pp. 282-287, 2013.

[19] CASARIN, S. J. Caracterização da temperabilidade de um aço C-Mn microligado ao boro, através de dilatometria e curvas de transformações de fases por resfriamento contínuo, Tese de D.Sc., EESC, São Carlos, 1996. 


\section{ORCID}

Hamilton Ferreira Gomes de Abreu

https://orcid.org/ 0000-0002-2293-837X

Marcelo José Gomes da Silva

https://orcid.org/0000-0003-1171-5944

Julianne Ribeiro dos Santos (ORCID ID https://orcid.org/0000-0002-8990-3243 